\title{
Separate Contributions of Kinematic and Kinetic Errors to Trajectory and Grip Force Adaptation When Transporting Novel Hand-Held Loads
}

\author{
Frederic Danion, ${ }^{1 *}$ Jonathan S. Diamond, ${ }^{2 *}$ and J. Randall Flanagan ${ }^{2,3}$ \\ ${ }^{1}$ Institut de Neurosciences de la Timone, UMR 7289, CNRS and Aix-Marseille University, 13385 Marseille cedex 5, France, and ${ }^{2}$ Centre for \\ Neuroscience Studies and 'Department of Psychology, Queen's University, Kingston, Ontario, K7L 3N6, Canada
}

\begin{abstract}
Numerous studies of motor learning have examined the adaptation of hand trajectories and grip forces when moving grasped objects with novel dynamics. Such objects initially result in both kinematic and kinetic errors; i.e., mismatches between predicted and actual trajectories and between predicted and actual load forces. Here we investigated the contribution of these errors to both trajectory and grip force adaptation. Participants grasped an object with novel dynamics using a precision grip and moved it between two targets. Kinematic errors could be effectively removed using a force channel to constrain hand motion to a straight line. When moving in the channel, participants learned to modulate grip force in synchrony with load force and this learning generalized when movement speed in the channel was doubled. When the channel was removed, these participants continued to effectively modulate grip force but exhibited substantial kinematic errors, equivalent to those seen in participants who did not previously experience the object in the channel. We also found that the rate of grip force adaptation did not depend on whether the object was initially moved with or without a channel. These results indicate that kinematic errors are necessary for trajectory but not grip force adaptation, and that kinetic errors are sufficient for grip force but not trajectory adaptation. Thus, participants can learn a component of the object's dynamics, used to control grip force, based solely on kinetic errors. However, this knowledge is apparently not accessible or usable for controlling the movement trajectory when the channel is removed.
\end{abstract}

\section{Introduction}

Numerous studies of motor learning have examined the adaptation of hand trajectories and grip force when transporting objects with novel dynamics specifying the mapping between applied force and motion (Shadmehr and Mussa-Ivaldi, 1994; Flanagan et al., 2003; Caithness et al., 2004; Nowak et al., 2004; Crevecoeur et al., 2010; Danion et al., 2012). Initially moving such objects results in kinematic errors, i.e., mismatches between predicted and actual hand trajectories, as well as kinetic errors, i.e., mismatches between predicted and actual load forces acting on the hand that are revealed by poor modulation of grip force with load force. However, with practice, people adapt so as to generate approximately straight-line hand movements and modulate grip force in synchrony with load force (Flanagan et al., 2003).

Although kinematic errors are considered to be critical for trajectory adaptation (Smith et al., 2006; Shadmehr et al., 2010; Melendez-Calderon et al., 2011; Wolpert et al., 2011), it is not known whether kinetic errors also contribute. Likewise, although

Received Aug. 7, 2012; revised 0ct. 16, 2012; accepted Nov. 17, 2012.

Author contributions: F.D., J.S.D., and J.R.F. designed research; F.D., J.S.D., and J.R.F. performed research; F.D.,

J.S.D., and J.R.F. analyzed data; F.D., J.S.D., and J.R.F. wrote the paper.

We thank Martin York, Sean Hickman, and Diane Fleming for technical support.

*F.D. and J.S.D. contributed equally.

Correspondence should be addressed to J. Randall Flanagan, Department of Psychology, Queen's University, Kingston, 0N, K7L 3N6, Canada. E-mail: flanagan@queensu.ca.

DOI:10.1523/JNEUROSCI.3772-12.2013

Copyright $\odot 2013$ the authors $\quad 0270-6474 / 13 / 332229-08 \$ 15.00 / 0$ it seems reasonable to assume that kinetic errors drive grip force adaptation, it is not known whether kinematic errors also contribute. Here we investigated the contributions of these errors to both trajectory and grip force adaptation. Importantly, the prediction of both load forces and hand trajectories relies on an internal model of object dynamics, and prediction errors can be used to update the internal model. A key question is whether kinematic and kinetic errors update a common internal model, or separate models used for trajectory and grip force adaptation, respectively.

In our task, participants grasped an object with a vertical precision grip and moved between targets in a horizontal plane. One robot, attached to the object, was used to create unusual object dynamics. A second robot, attached to the wrist, could implement a force channel that constrained hand motion to a straight line, effectively removing kinematic errors that would otherwise result from the unusual object dynamics (Scheidt et al., 2000; Smith et al., 2006). This setup enabled us to compare grip force adaptation with and without kinematic errors as well as test whether experiencing kinetic errors in the channel would benefit trajectory adaptation when the channel was subsequently removed.

If kinematic and kinetic errors update a common internal model of object dynamics, we would expect kinetic errors experienced in the channel to benefit trajectory control when subsequently moving without the channel. Moreover, we would expect grip force adaptation to be quicker when initially experiencing 
A

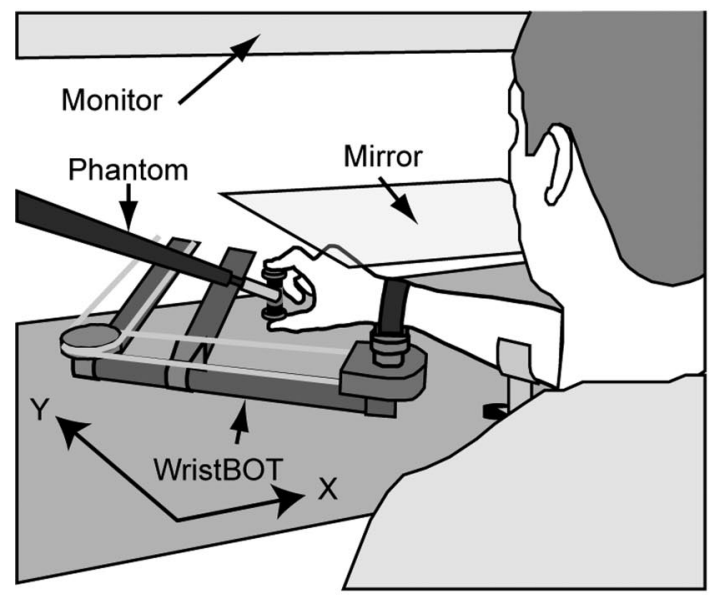

B

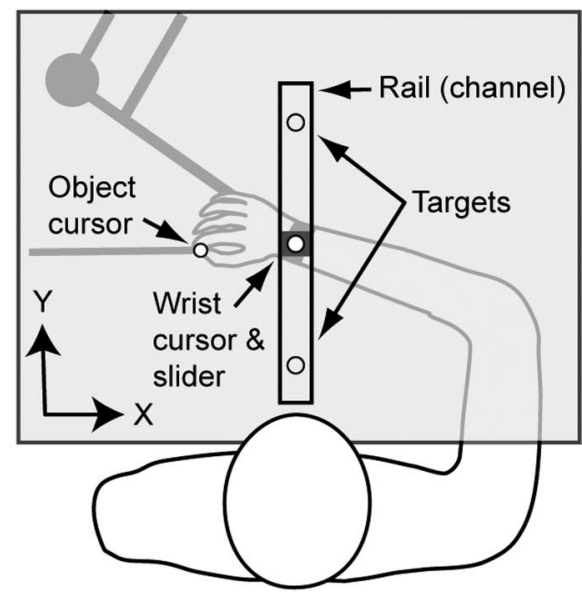

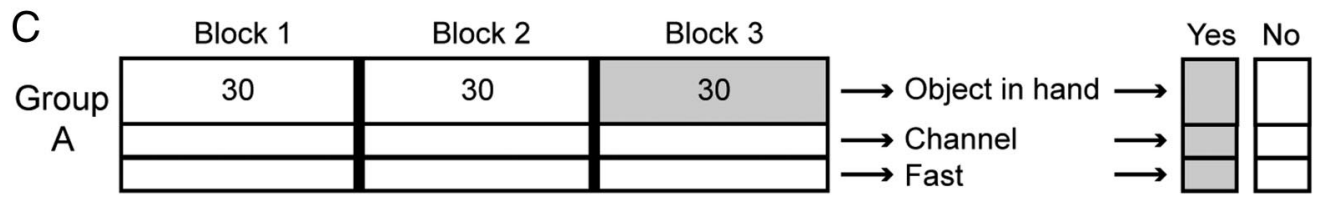
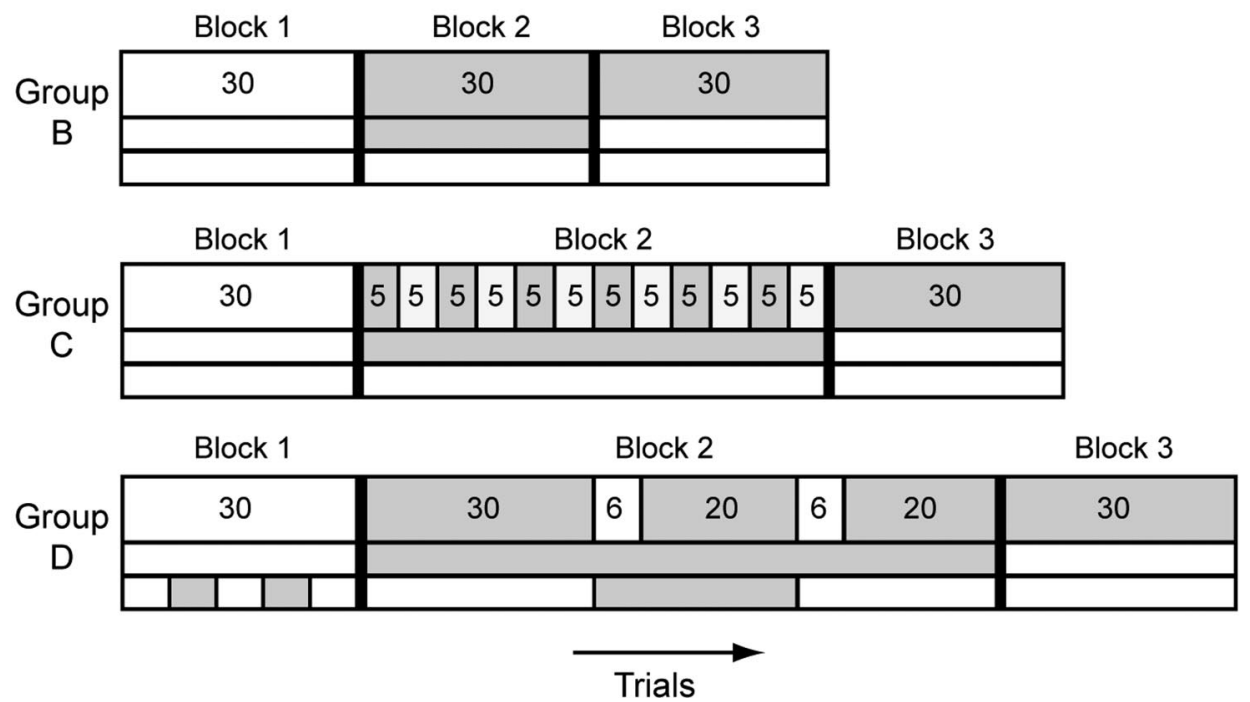

Figure 1. The apparatus and experimental design. $\boldsymbol{A}, \boldsymbol{B}$, Schematic drawings of the experimental setup. $\boldsymbol{C}$, Experimental design for each group of participants (see Materials and Methods for further information).

novel object dynamics without, compared to with, the channel because both kinematic and kinetic errors would update the internal model, as opposed to kinetic errors alone. However, if kinematic and kinetic errors update separate internal models, we would not expect kinetic errors experienced in the channel to benefit subsequent trajectory control. Moreover, we would not expect grip force adaptation to be faster when first experiencing the novel object dynamics without the channel because kinematic errors experienced without the channel would not contribute to updating the internal model used for grip force control.

\section{Materials and Methods}

Participants. Forty-five participants (21 women) were recruited from the population of undergraduate and graduate students at Queen's University. Participants provided written informed consent and received monetary compensation for their time. The ethics committee of Queen's
University approved the study. The participants were randomly assigned to one of four groups, with 11 participants in Groups A, B, and C and 12 participants in Group D (Group A: age $=22.0 \pm 2.4$ years, height $=$ $1.72 \pm 0.09 \mathrm{~m}$, mass $=73.2 \pm 15.5 \mathrm{~kg}$; Group B: age $=23.3 \pm 4.6$ years, height $=1.75 \pm 0.11 \mathrm{~m}$, mass $=82.4 \pm 3.5 \mathrm{~kg}$; Group C: age $=22.9 \pm 4.9$ years, height $=1.70 \pm 0.07 \mathrm{~m}$, mass $=67.6 \pm 17.0 \mathrm{~kg}$; Group D: age $=$ $20.8 \pm 2.3$ years, height $=1.69 \pm 0.08 \mathrm{~m}$, mass $=61.8 \pm 7.3 \mathrm{~kg}$ ). All of the participants reported that they were right-handed.

Apparatus. While seated, participants moved a circular cursor (diameter $20 \mathrm{~mm}$ ) representing the position of the right wrist between two circular targets (diameter $20 \mathrm{~mm}$ ) presented in a horizontal plane (Fig. $1 A, B)$. The two targets were aligned in the participant's midsagittal plane ( $y$-axis) and separated by $15 \mathrm{~cm}$. The wrist was strapped to the endpoint of a planar robotic manipulandum (WristBOT, Howard et al., 2009) that measured the position of the wrist (resolution $0.1 \mathrm{~mm}$ ) and that could apply forces in the horizontal plane. In some trials, participants were required to grasp, with the right hand, a cylindrical object using a preci- 
sion grip with the tips of the index finger and thumb contacting vertically aligned horizontal circular surfaces (diameter $25 \mathrm{~mm}$ ) located on the top and bottom (64 $\mathrm{mm}$ apart), respectively. In these trials, a circular cursor (diameter $10 \mathrm{~mm}$ ) representing the position of the object in the horizontal plane was displayed (Fig. 1B). Each grip surface was mounted on a six-axis force transducer (Nano F/T, ATI Industrial automation) that measured applied forces $(0.05 \mathrm{~N}$ resolution $)$ and torques in three dimensions. The grip surfaces were covered with sandpaper and were free to spin about the long axis of the object. The object was attached to a lightweight robotic manipulandum (Phantom Haptic Interface, 3.0, Sensable Devices) via a joint that allowed rotation about all axes except the long axis of the object. Thus, the combination of this joint and the spinning grip surfaces allowed free rotation of the object in three dimensions. Three optical encoders, placed on the three motors of the manipulandum, measured the object's position in three dimensions $(0.1 \mathrm{~mm}$ resolution).

In some trials, a force channel, created with the WristBOT manipulandum, constrained the motion of the wrist to a straight line between the two targets (Fig. 1B). The force channel was implemented as a damped spring (with a stiffness of $5 \mathrm{~N} / \mathrm{mm}$ and a viscosity of $0.01 \mathrm{~N} / \mathrm{mm} / \mathrm{s}$ ) that applied forces to the wrist perpendicular to the straight line between the targets if the wrist cursor veered off this straight line. In addition, the ends of the channel were capped such that the wrist cursor could not move $>25 \mathrm{~mm}$ beyond the target centers. In force channel trials, a rectangle (width of $30 \mathrm{~mm}$ and length of $200 \mathrm{~mm}$ ) was displayed representing the walls of the channel (Fig. 1B). When moving in the channel, the cursor representing the wrist was positioned in the center of a visual slider (square of width $30 \mathrm{~cm}$ ) that moved along the length of the channel. In channel trials, we displayed the position of the wrist projected onto the line between the targets. In other words, very small deviations of the wrist cursor off the line between the targets were not displayed such that the wrist cursor was always in the center of the channel.

A visual display system was used to present the two targets, the cursors representing the wrist and object, and the walls of the force channel in a horizontal plane at the height of the wrist. This system consisted of a 30 -inch monitor positioned horizontally above a mirror located half way between the monitor and plane of wrist movement (Fig. 1A). Participants viewed the visual scene displayed on the monitor via the mirror, which blocked vision of the arm and grasped object.

Procedure. A single trial involved moving the wrist cursor between the two targets $($ Fig. $1 B$ ). In all cases, participants generated alternating outward and inward movements in successive trials. In a given trial, the movement could be generated either while grasping the object (in which case the cursor representing the object was displayed) or without the object in hand. In addition, in a given trial the force channel could either be on (in which case the rectangle representing the channel was displayed) or off. In trials in which the object was grasped, the forces generated by the object on the fingertips depended on object velocity as follows:

$$
\left[\begin{array}{l}
f_{x} \\
f_{y}
\end{array}\right] N=\left[\begin{array}{cc}
0 & -0.012 \\
0 & 0
\end{array}\right] \frac{N}{\mathrm{~mm} / \mathrm{s}}\left[\begin{array}{c}
\dot{x} \\
\dot{y}
\end{array}\right] \mathrm{mm} / \mathrm{s}
$$

Thus, the object generated a velocity-dependent lateral force ( $x$-axis in Fig. $1 B$ ) on the fingertips proportional to the velocity of the object in the straight-ahead direction ( $y$-axis in Fig. 1B). The direction of the lateral force depended on the direction of object movement such that a leftward force was applied when the object was moved outwards (from the near target to the far target) and a rightward force was applied when the object was moved inwards (from the far target to the near target).

Before a trial could be initiated, the participant had to position the center of the wrist cursor within $5 \mathrm{~mm}$ of the center of the current start target for $200 \mathrm{~ms}$. For the purpose of providing feedback about movement duration, movement onset was defined at the time at which the wrist velocity first exceeded $30 \mathrm{~mm} / \mathrm{s}$. In standard speed trials, the current end target changed color (from cyan to white) $400 \mathrm{~ms}$ after movement onset, and participants were asked to position the wrist cursor at the end target at the time at which the end target changed color. In fast speed trials, the duration was $200 \mathrm{~ms}$.
The sequences of trials experienced by the four groups of participants are illustrated in Figure 1C. Each group completed 3 blocks of trials. For all groups, block 1 consisted of 30 practice trials ( 15 outward and 15 inward movements interleaved) without the object and without the force channel. The primary aim of these trials was to allow participants to practice moving between the targets in the prescribed duration. Participants in Groups A, B, and C performed these movements at the standard speed whereas participants in Group D performed alternating sets of standard and fast speed trials, with 6 trials per set, starting and ending with standard speed trials. These participants were told about the required speed at the start of each set. For all groups, block 3 consisted of 30 movements with the object in hand and without the force channel. The primary aim of the current study was to test whether moving the grasped object with the force channel (in block 2) would facilitate adaptation of movement trajectories when moving the object without the channel.

The four groups differed in terms of the trials they experienced in block 2. Participants in Group A, who served as controls, performed 30 trials without the object and without the force channel (as in block 1). Participants in Group B performed 30 trials with the object in hand and with the force channel. Participants in Group C performed alternating sets of 5 trials with and without the object in hand and with the force channel turned on throughout. This group was included in an effort to increase the salience of the forces generated by the object. Participants in Group D first performed 30 standard speed trials with the object and force channel. They then completed 6 fast speed trials in the force channel without the object, 20 fast speed trials in the force channel with the object, 6 standard speed trials in the force channel without the object, and 20 standard speed trials in the force channel with the object. The group was included to determine whether grip force adaptation, expected over the first 30 standard speed trials, generalized across movement speeds. The 6 no-object trials at the start of each change in required movement speed were included to ensure that participants were moving at the correct speed when first transporting the object at the new speed.

As described above, participants in Groups B, C, and D first experienced the object with the motion of the wrist constrained by the force channel. To make the forces generated by channel versus the object as distinct as possible, all of these participants completed three channel exploration trials, without the object in hand, between blocks 1 and 2 . Participants were told that the channel would act like a rail to which their wrist was attached (via the slider) and that they could move their wrist along the rail but not off the rail. To initiate the first exploration trial, participants were required to position the wrist cursor at a start position corresponding to the near target. The rectangle representing the walls of the channel and the slider were then displayed and the channel was turned on. Participants were given $10 \mathrm{~s}$ to explore the effects of the channel by moving along it and generating forces perpendicular to it. Participants were then given two additional $10 \mathrm{~s}$ trials to explore the channel. Following these exploration trials, participants were asked to move the wrist cursor to the near start position located within the channel and the object was handed to them. The object cursor was visible as the object was handed to the participant so that they could see it approaching. They were told to grasp the object with a precision grip and to keep the orientation of the grip axis vertical. Participants were told that the object would generate forces on their fingertips when it was moved but that their wrist would be secured to the rail. Participants were also informed that they would be required to transport the same object without the help of the channel following the current block of channel trials. At this point, block 2 was initiated.

Participants in Groups B, C, and D also completed a similar transition trial between block 2 (with the channel) and block 3 (without the channel). Specifically, following the last trial of block 2, participants were asked to move the wrist cursor to the start position and the object was then taken from them and moved such that the object cursor was out of view. The rectangle representing the walls of the channel and the slider were then removed from view and participants were told that the channel had been removed. Participants were then given $10 \mathrm{~s}$ to move around the workspace as they wished so that they could appreciate that the channel had been removed. Participants then returned the wrist cursor to the start position and the object was handed back to them. Participants were 
told that they would now be required to make movements between the targets while holding the object and with the channel removed.

One of the two experimenters who were always present monitored the orientation of the object during object trials and encouraged the participant to keep the orientation of the grip axis vertical. The orientation of the object was also assessed during data analysis (see below). Participants were debriefed after completing the experiment. They were asked to comment on the forces exerted by the object both when moving in the channel and when subsequently moving without the channel. They were also invited to provide any additional comments.

Analysis. Force signals from the force sensors and position signals from the robotic devices (indicating the positions of the object and wrist) were recorded at $1000 \mathrm{~Hz}$ and digitally smoothed using a fourth-order, zero-phase lag Butterworth filter with a cutoff frequency of 14 Hz. A first order central difference equation, applied to the smoothed position signals, was used to compute the $x$ and $y$ velocities of the object and wrist. Grip force was computed as the average of the normal forces, applied by the index finger and thumb, at the two grip surfaces. Load force was computed as the sum of the tangential forces at each grip surface (i.e., the load force at each surface).

To assess whether the object was held in a vertical orientation, for each trial we computed the correlation between the load forces exerted by the index finger and thumb. In general, these forces were very similar, yielding an average correlation of 0.97 . Trials in which the correlation was $<0.50$ were removed from the analysis of grip force adaptation. This resulted in the removal of $2.6 \%$ of the trials.

To assess the coupling between grip force and load force we computed the correlation coefficient (at zero phase lag) between the two forces. To quantify the trajectory of the wrist, we computed the peak-to-peak lateral deviation of the wrist path. That is, we determined the difference between the largest and smallest $x$ positions observed during the movement (see $x$ coordinates in Fig. 1B). Movement onset was taken as the time at which the wrist velocity exceeded $20 \mathrm{~mm} / \mathrm{s}$, and movement offset was taken as the time at which wrist velocity went below $20 \mathrm{~mm} / \mathrm{s}$.

To quantify grip force and trajectory adaptation, we fit an exponential of the form $y=a e^{b x}+c$ to the grip-load force cross-correlation coefficients and the lateral trajectory deviations. We tested for the effects of group on all three parameters of the exponential using nonlinear regression. A 0.05 significance threshold was used for all analyses.

\section{Results}

\section{Grip force adaptation in the channel}

Figure $2 A$ illustrates grip and load forces and movement kinematics from the first trial in the force channel (block 2) for a single participant from Group B. Note that the load force scaled with the velocity of the object in the $y$ direction (i.e., along the channel). As expected, the coupling between grip force and load force on this initial trial with the object in hand was poor, yielding a correlation coefficient of 0.52 . This indicates that the participant failed to predict the load force (and modulate grip force appropriately). The motion path of the wrist was constrained to a straight-line path between the two targets. The path of the object was also quite straight. However, a small amount of curvature occurred due to the load force applied to the fingertip. Because

\section{B}
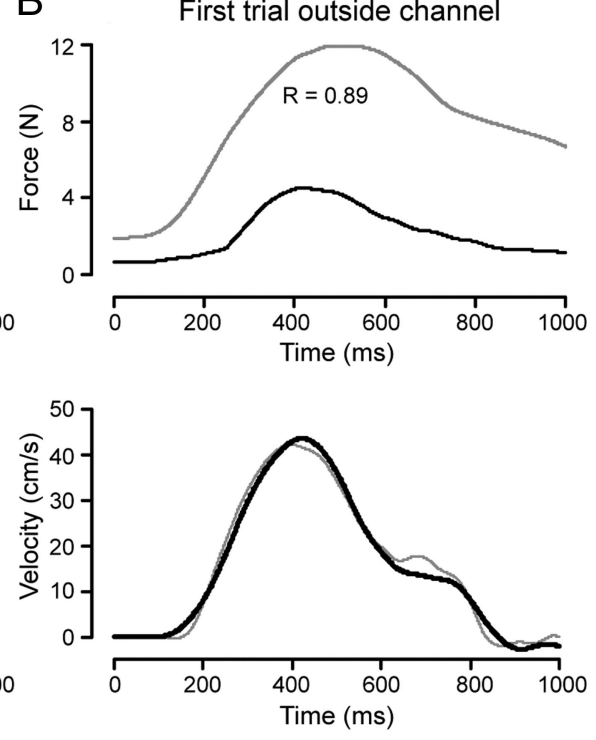

- Object path

- Wrist path

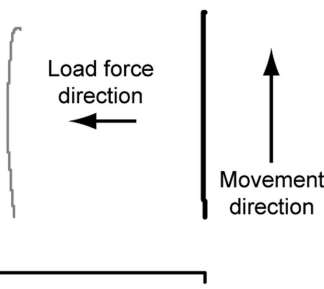

X Position
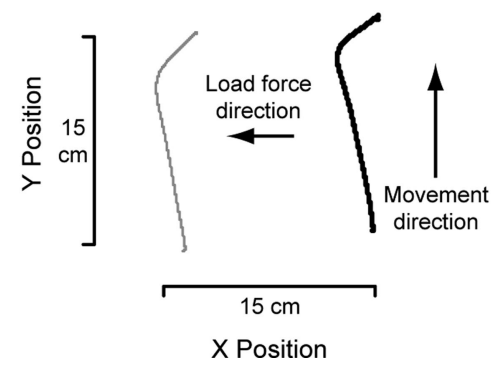

X Position

Figure 2. Representative trials. $A$, First trial while moving the unfamiliar object in the channel. $B$, First trial, after prior experience in the channel, while moving the same object without the channel. The same participant, from Group B, performed both trials. Note that although arm and object movements were relatively straight in $A$, the coupling between grip force and load force was weak. Conversely, in $\boldsymbol{B}$, movements were altered but the coupling between grip force and load force was strong.

the load force acted orthogonal to the direction of movement, the velocity profiles of the wrist and object were similar to what one would expect without a load or with a standard inertial load; i.e., they were approximately bell-shaped (Morasso, 1981; Ruitenbeek, 1984; Atkeson and Hollerbach, 1985; Hogan and Flash, 1987; Flanagan and Wing, 1997).

The average maximum absolute lateral deviation of the wrist, based on means from all participants in Groups B and C, was 2.7 $\mathrm{mm}(\mathrm{SE}=0.2 \mathrm{~mm})$. Thus, the channel was effective in maintaining an approximately straight-line wrist path. However, we found that the maximum absolute lateral deviation of the wrist increased slightly over the first 10 trials across trials performed in the channel and then remained steady. The average deviation was significantly greater $(p<0.05)$ over the last two trials combined $[$ mean $(\mathrm{M})=3.3 \mathrm{~mm}, \mathrm{SE}=0.32 \mathrm{~mm}$ ] than over the first two trials combined $(\mathrm{M}=1.2 \mathrm{~mm}, \mathrm{SE}=0.08)$. This indicates that participants learned to exploit the channel, allowing forces applied by the channel to counteract the load force applied by the object. The average maximum absolute lateral deviation of the grasped object, based on means from all participants in Groups B and $\mathrm{C}$, was $15.7 \mathrm{~mm}(\mathrm{SE}=0.8 \mathrm{~mm})$. Thus, on average, the maximum lateral motion of the object relative to the wrist, which in the direction of the load applied by the object to the hand, was $\sim 13 \mathrm{~mm}$. Although the load acting on the object slightly perturbed the trajectory of the object and hand when moving in the channel, as will be shown below, this perturbation was much 


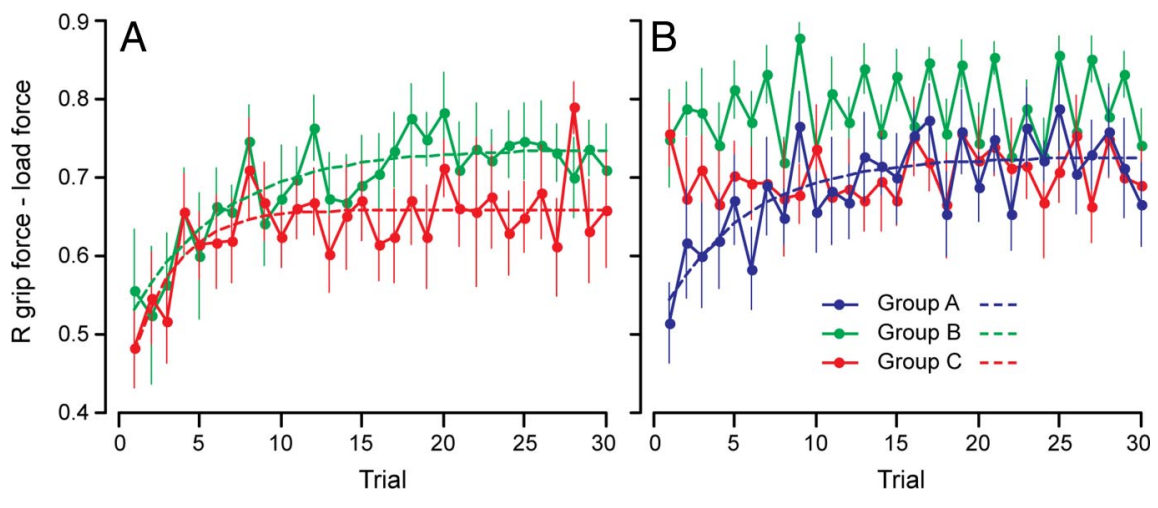

Figure 3. Mean grip-load force coupling as a function of trial and group. $\boldsymbol{A}$, Average correlation coefficients between grip force and load force while experiencing the object in the channel. $\boldsymbol{B}$, Average correlation coefficients between grip force and load force when moving the object without the channel. Dashed lines represent exponentials fit to the data. The comparison between the 3 groups indicates that prior experience moving in the channel (Groups B and C) benefited grip-load coupling. Error bars correspond to 1 SE.

smaller than that observed in participants who first experienced the load without the channel.

Figure $3 A$ presents the evolution of the correlation coefficient $(R)$ between grip force (GF) and load force (LF) over successive object transport trials performed in the channel (block 2) by participants from Groups B and C. In both groups, as the number of trials increased, the correlation between GF and LF increased. To assess grip force adaptation, we fit exponentials to both groups. For each group, all three parameters of the exponential were significantly different $(p<0.05)$ than zero. Nonlinear regression revealed no significant differences between Groups B and $C$ in terms of the leading value $(-0.243$ vs -0.261$)$ or the learning rate $(-0.175$ vs -0.370$)$. However, the asymptote was significantly greater $(p<0.05)$ for Group B $(0.737)$ than for Group $C(0.660)$. These results indicate that participants in Groups B and C learned to modulate grip force in synchrony with load force while moving the grasped object in the channel.

\section{Transfer of grip force adaptation outside the channel}

Figure $2 B$ illustrates grip and load forces and movement kinematics from the first trial with the force channel removed (block 3 ) for the same participant shown in Figure $2 A$. In contrast to the first trial performed in the channel (block 2; Fig. 2A), good coupling between grip force and load force was seen in the first trial outside the channel, yielding a correlation coefficient of 0.89 . Importantly, the path of the wrist (as well as the path of the object) was strongly deflected by the load force applied to the object. This required the participant to generate arm movement correction, which is also evident in the $y$ velocity profiles of the wrist and object. Thus, whereas predictive grip force control was maintained when the channel was removed, the participant was seemingly unable to exploit knowledge of object dynamics, gained when moving in the channel, to compensate for the effects of the load on the movement trajectory.

Figure $3 B$ presents the evolution of the correlation coefficient between grip force and load force over successive trials outside the channel (block 3) for Groups A, B, and C. When the channel was removed, participants from Groups B and C were able to maintain good coupling between grip force and load force, as indicated by relatively high correlation coefficients observed in the initial trials. This view was confirmed by a two-way ANOVA that compared mean $R$ values over the 4 initial trials performed outside the channel and the mean $R$ values over the last 4 trials performed in the channel for both Group $\mathrm{B}$ and $\mathrm{C}$. There were no main effects of GROUP or TRIAL BLOCK (first 4 vs last 4 ) and no interaction $(p>0.22$ in all three cases), indicating that both groups had no difficulties in maintaining accurate grip force control when the channel was removed (block 3 ). In contrast, participants from Group A exhibited lower $R$ values during initial trials. A one-way ANOVA examining the first trial outside the channel revealed that the $R$ value was smaller in Group A than in Groups B and C combined $(p<0.02)$. Thus, in terms of grip force control, participants from Groups B and $\mathrm{C}$ appeared to benefit from prior exposure to object dynamics in the channel (block 2). However, due to the nature of the load acting on the object, good transfer of grip force control would be expected if participants keep modulating grip force in the same way once the channel is removed in block 3 .

For Group A, $R$ values improved across trials in block 3 in much the same way that they improved for Groups B and $C$ when moving in the channel (block 2). Fitting an exponential to the $R$ values for Group A revealed that all three parameters $(\mathrm{a}=0.221$, $\mathrm{b}=0.192, \mathrm{c}=0.726)$ were significantly different from zero $(p<$ 0.05 ). Nonlinear regression was used to compare the exponential fit to the $R$ values of Group A (outside the channel; block 3 ) with the exponentials fit to the $R$ values for Groups $\mathrm{B}$ and $\mathrm{C}$ when moving inside the channel (block 2). This analysis failed to reveal significant differences among the three groups in terms of the leading value and learning rate. The asymptote was greater for Group B than Group A $(p<0.05)$ but there was no difference between Group C and Group A. Thus, overall the adaptation of grip force control did not seem to benefit from kinematic errors associated with arm movements without the channel.

\section{Trajectory adaptation outside the channel}

A central aim of the current study was to assess whether prior exposure to object dynamics in the channel would benefit trajectory adaptation when the channel was removed. Figure 4 shows the peak-to-peak lateral deviation of the wrist path as a function of trial in block 3 for Groups A, B, and C. As expected, for participants in Group A, who were experiencing the novel dynamics for the first time, the wrist path was substantially deviated in initial trials. However, these participants gradually adapted to the perturbation over $\sim 15-20$ trials. As can be appreciated visually in Figure 4, participants in Groups B and C did not seem to benefit from prior exposure to object dynamics when moving in the channel (block 2). That is, the lateral deviation functions observed for these groups were similar to that observed for group A. To quantify trajectory adaptation, we fit exponentials to the lateral deviations for each group. For each of the three groups, all three parameters of the exponential were significantly different $(p<0.001)$ than zero. Nonlinear regression revealed no significant differences $(p>0.05)$ among the three groups in terms of the leading value (37.1, 47.2, and 45.0 for Groups A, B, and C), the learning rate $(-0.280,-0.259$, and -0.335$)$, or the asymptote $(15.63,17.92$, and 16.67). Thus, this analysis indicates that all three groups exhibited similar trajectory adaptation and that there was no benefit of prior exposure to object dynamics in the channel. 


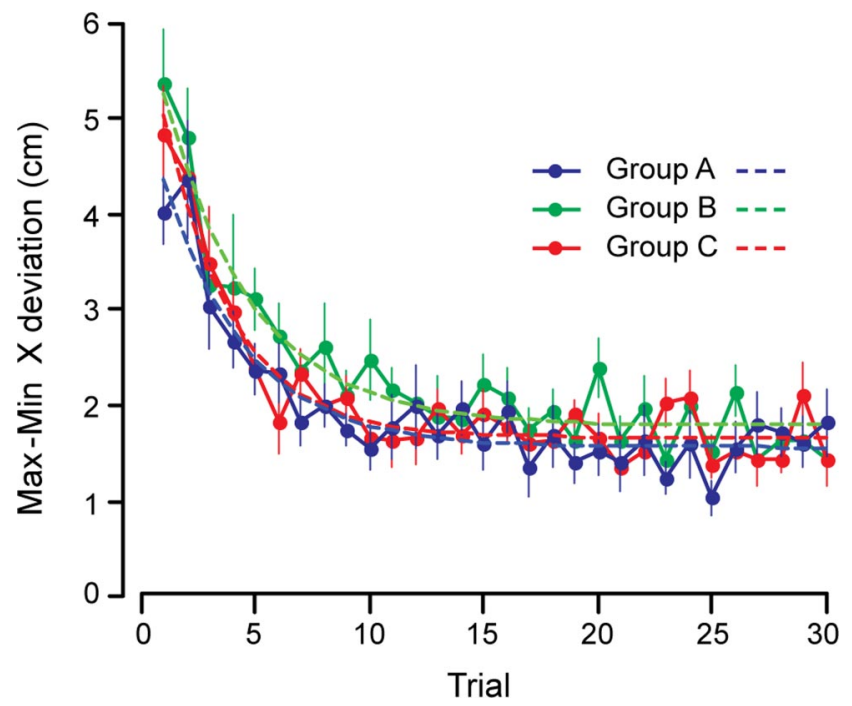

Figure 4. Mean peak-to-peak lateral deviation of the wrist, as a function of trial and group, when moving without the channel. The dashed lines represent exponentials fit to each series of data points. Error bars correspond to $1 \mathrm{SE}$.

\section{Generalization across speeds within the channel}

The fact that participants in Groups B and C adapted their grip force to the novel load force experienced in the channel (block 2) suggest that they learned that this load was velocity dependent. To evaluate whether this is the case, we ran an additional group of participants (Group D) who manipulated the object at various speeds in the channel (block 2). These participants first performed 30 movements at the same speed as participants from Groups B and C and were then instructed to double their movement speed for 20 trials before completing 20 trials at the initial, regular speed.

The instruction to double movement speed led to an approximate doubling of the magnitude of peak LF. Overall, the mean load force peak was $4.8 \pm 0.3 \mathrm{~N}$ (average based on participant means $\pm \mathrm{SE}$ ) during the first 30 trials, then reached $8.2 \pm 0.4 \mathrm{~N}$ during the subsequent 20 faster trials, and finally decreased to $5.1 \pm 0.3 \mathrm{~N}$ during the last 20 trials performed at normal speed. One-way ANOVA showed significant variation in the load force peak among the three sessions $(p<0.001)$. Post hoc analyses confirmed that load force peaks during the intermediate session were greater than in the first and last sessions $(p<0.001)$, but that there was no significant difference between the load force peaks in the first and the last sessions $(p=0.29)$. Note that transitions in peak load force across sessions were brisk. Indeed the peak load during the very first trial performed at fast speed was $7.8 \mathrm{~N}$, a value very close from the overall mean of the trials. Similarly, the peak load force dropped immediately to $4.6 \mathrm{~N}$ during the first trial of the last session. These immediate transitions were possible because our protocol included practice trials without the object at the start of each session so that participants moved at the intended speed when first moving the object.

To assess generalization in grip-load force coupling across movement speeds, we focused on the correlation coefficient in the first trial and the last four trials performed at each speed (i.e., the initial regular speed, the fast speed, and the second regular speed). Figure 5 shows the mean correlation coefficients averaged across participants for each of these three sessions. As expected, the correlation between grip force and load force was relatively poor in the first trial performed with the object (session 1) and the $R$ value was similar to the initial $R$ values observed for Groups

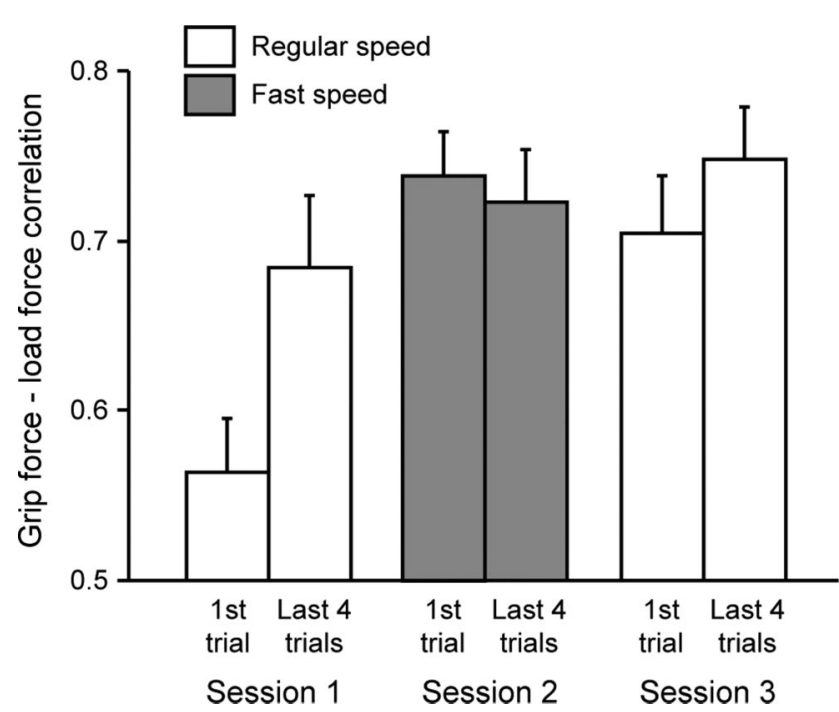

Figure 5. Mean grip-load force coupling as a function of trials for Group D when moving in the channel. The correlation between grip force and load force is presented at the beginning and end of each session (each one requiring a different movement speed). Note that grip-load force coupling generalized well across movement speeds. Error bars correspond to $1 \mathrm{SE}$.

B, C, and D (Fig. 3). Two-way ANOVA showed a TRIAL by SESSION interaction $(p<0.05)$. Post hoc analyses of the interaction revealed that $R$ values during the first trial in session 1 were significantly smaller than all the other $R$ values $(p<0.01$ in all 5 cases), and that there were no significant differences between the remaining five $R$ values. This indicates that the good coupling between grip force and load force, observed at the end of session 1 , did not deteriorate with successive changes in movement speed. This suggests that, once updated, predictive grip force control generalized well across movement speeds.

When the channel was subsequently removed (block 3), participants of Group D were able to maintain a good coupling between grip force and load force $(r=0.73$ in the first trial without the channel), as previously shown for participants of Groups $\mathrm{B}$ and $\mathrm{C}$. To assess whether the coupling of grip force and load force changed over trials performed outside the channel, we compared $R$ values averaged over the first 4 and last 4 trials. A two-way BLOCK (first 4 versus last 4 trials) by GROUP (Groups B, C, and D) ANOVA failed to show main effects of BLOCK $(p=0.57)$ or GROUP $(p=0.40)$, and there was no interaction $(p=0.61)$. This indicates that all 3 groups maintained efficient grip force control throughout the trials performed without the channel.

In terms of trajectory control when the channel was removed (block 3), participants from Group D did not benefit from exposure to object dynamics in the channel. All three parameters of the exponential fit to the lateral deviations for Group D (leading value $=36.78$, learning rate $=-0.180$, asymptote $=18.91)$ were significantly different from zero, thereby confirming the reduction of lateral deviations across trials. Nonlinear regression comparing all four groups revealed that the asymptote was slightly but significantly greater for Group D than Group A $(p<0.05)$ and that the learning rate was slightly but significantly slower for Group D than Group C $(p<0.05)$. No other significant differences between groups were observed. Thus, experiencing the novel object dynamics at different movements speeds in the channel clearly did not facilitate trajectory adaptation after the channel was removed. 


\section{Discussion}

The goal of this study was to investigate the contribution of kinematic and kinetic errors to grip force and trajectory adaptation when learning to manipulate objects with unusual dynamics. To achieve this goal, we developed a novel setup that enabled us to compare grip force adaptation with and without kinematic errors and to test whether experiencing kinetic errors in the channel benefits trajectory control when subsequently moving without the channel. We found that participants successfully adapted their grip force when moving in the channel (Groups B, C, and D, block 2) and that grip force adaptation was no quicker when initially experiencing the object without the channel (Group A, block 3). We also found that prior experience moving the object in the channel did not benefit trajectory adaptation when moving without the channel.

\section{Updating and generalization of grip force control}

Previous studies (Flanagan et al., 2003; Nowak et al., 2004; Danion et al., 2012) have shown that people can learn to modulate grip force in synchrony with load force when manipulating objects with unfamiliar dynamics. In all of these studies, both kinetic and kinematic errors were available and thus the separate contributions of these errors could not be assessed. Our finding that grip force control can be updated when moving in the channel (Groups B, C, and D, block 2) and thus in the absence of significant kinematic errors, suggests that kinetic errors are sufficient for driving grip force adaptation. Although kinematic errors, in principle, can provide information about object dynamics, our finding that grip force adaptation was no quicker when first experiencing the object without the channel (Group A, block 3) suggests that these errors are not key inputs for grip force control. This conclusion fits well with observations showing that tactile information at the fingertips dominates over proprioceptive information in terms of controlling grip force responses to unexpected load force perturbations (Cole and Abbs, 1988; Häger-Ross and Johansson, 1996; see also Danion, 2007).

We found that learning to modulate grip force in synchrony with load force, when moving in the channel, generalized across movement speeds (Group D, block 2). This result suggests that participants did not simply learn to compensate for a specific temporal profile of load force, but rather acquired an internal model capturing the object dynamics. Previous studies have shown that trajectory adaptation to novel, velocity-dependent loads also generalizes across movement speeds (Goodbody and Wolpert, 1998; Joiner et al., 2011). Thus, it appears that grip force adaptation, which seems to be driven by kinetic errors, and trajectory adaptation, thought to be driven by kinematic errors, both involve the acquisition of an internal model of object dynamics.

\section{Updating of arm movement control}

Although the grip force results showed that participants learned a component of the object's dynamics when moving in the channel (Groups B, C, and D, block 2), this knowledge was apparently either not relevant or not accessible when subsequently controlling the motion of the hand and object without the channel. Although we cannot rule out the possibility that kinetic errors contribute to trajectory adaptation when combined with kinematic errors, our results indicate that kinetic errors alone are insufficient to drive learning that can be exploited for trajectory control. Instead, kinematic errors appear to be necessary for trajectory adaptation.
Previous studies of arm movement adaptation to novel handheld loads have shown that people primarily attribute the load to the object such that relatively small after-effects are observed when the object is released from grasp (Lackner and DiZio, 2005; Cothros et al., 2006; Berniker and Kording, 2008; Kluzik et al., 2008). One possible explanation for our results is that, despite our efforts to provide explicit contextual information about the channel, object, and perturbation (see Materials and Methods), participants failed to link the load forces experienced at the fingertips to the object when moving in the channel. However, the fact that participants continued to modulate grip force in synchrony with load force on the first trial after the channel was removed (Groups B, C, and D, block 3) indicates that they expected the same object dynamics, at least at the level of grip force control. Interestingly, when participants were debriefed after the experiment, they indicated that the forces applied by the object when moving in the channel were much weaker that those experienced when the channel was removed and were generally unable to describe the forces experienced in the channel (i.e., that they scaled with movement speed and were leftward and rightward for outward and inward movements, respectively). This suggests that the grip force adaptation was based on implicit rather than explicit processes (see Mazzoni and Krakauer, 2006 for a discussion of these processes). Previous work suggests that people can use cognitive strategies, based on explicit knowledge of kinematic errors experienced when moving a novel load in one context, to compensate for the same load experienced in a new context (Criscimagna-Hemminger et al., 2003; Malfait and Ostry, 2004). Had our participants been able to explicitly appreciate the kinetic errors experienced in the channel, it is possible that they could have developed similar strategies to generate compensatory arm motor commands when the channel was removed.

One interpretation of our results is that participants relied on distinct internal models of object dynamics for grip force and trajectory control. We previously suggested that grip force adaptation involves rapid learning of a forward model mapping arm motor commands onto predicted load forces, whereas trajectory adaptation involves slower learning of an inverse model mapping desired movements onto arm motor commands (Flanagan et al., 2003). However, a limitation of that study is that grip force and trajectory control were measured using different dependent variables, making the comparison of learning rates problematic. The current study does not suffer from this limitation and it therefore provides more convincing evidence that distinct neural processes drive grip force and trajectory adaptation. This possibility was raised in a recent study that investigated the ability of participants to move a manipulandum while controlling explicitly the contact forces at the handle (Chib et al., 2009). Based on the observation that transcranial magnetic stimulation over the parietal cortex selectively disrupted arm motion control but not the control of contact forces, the authors argued that object manipulation involves independent control of hand motions and interaction forces between the object and hand.

In most studies of arm movement adaptation to novel loads, the load is applied to the hand via a vertical handle that is grasped in a power grip (Shadmehr and Mussa-Ivaldi, 1994; Malfait et al., 2002; Davidson et al., 2005; Diedrichsen et al., 2005; Lackner and DiZio, 2005; Izawa et al., 2008; Shadmehr et al., 2010). It is an open question whether such adaptation, which is thought to be driven by kinematic errors, would benefit grip force control when subsequently moving the load using a precision grip. It has been shown that adapting arm movements to novel object dynamics while seated, enables participants to appropriately scale anticipa- 
tory postural adjustments when subsequently performing the same movements in a standing position (Ahmed and Wolpert, 2009). Because grip force adjustments can be viewed as postural adjustments that compensate for forces arising from arm movements (Wing et al., 1997), we might expect that learning object dynamics when using a power grip would benefit grip force control when subsequently using a precision grip.

\section{Concluding comments}

Object manipulation and transport tasks are among the most widely used to study how people learn novel mappings between their motor commands and their behavioral consequences (Flanagan et al., 2006; Shadmehr et al., 2010; Wolpert et al., 2011). Whereas many studies have focused on the adaptation of arm movement trajectories, relatively little attention has been paid to the adaptation of grip force control, which is a key component of skilled object manipulation (Johansson and Flanagan, 2009). Our results further document the crucial role of kinematic errors for arm movement adaptation (Smith et al., 2006; Shadmehr et al., 2010; Wolpert et al., 2011), but also highlight the key role of kinetic errors for grip force adaptation, as well as the ability of grip force to generalize across multiple contexts. More generally, these results suggest that arm movement control and grip force control are mediated by distinct internal representations of object dynamics. Previous studies have shown that learning of dynamics is state-dependent such that adaptation in one movement direction or orientation of the object in hand shows limited generalization to other directions or orientations (Shadmehr and Moussavi, 2000; Thoroughman and Shadmehr, 2000; Mah and Mussa-Ivaldi, 2003; Ingram et al., 2010, 2011). Our results suggest that such learning also depends on the motor response required to compensate for the dynamics. That is, learning of object dynamics associated with adapting grip force commands in the channel does not generalize to the control of arm motor commands when the channel is removed.

\section{References}

Ahmed AA, Wolpert DM (2009) Transfer of dynamic learning across postures. J Neurophysiol 102:2816-2824. CrossRef Medline

Atkeson CG, Hollerbach JM (1985) Kinematic features of unrestrained vertical arm movements. J Neurosci 5:2318-2330. Medline

Berniker M, Kording K (2008) Estimating the sources of motor errors for adaptation and generalization. Nat Neurosci 11:1454-1461. CrossRef Medline

Caithness G, Osu R, Bays P, Chase H, Klassen J, Kawato M, Wolpert DM, Flanagan JR (2004) Failure to consolidate the consolidation theory of learning for sensorimotor adaptation tasks. J Neurosci 24:8662-8671. CrossRef Medline

Chib VS, Krutky MA, Lynch KM, Mussa-Ivaldi FA (2009) The separate neural control of hand movements and contact forces. J Neurosci 29:3939-3947. CrossRef Medline

Cole KJ, Abbs JH (1988) Grip force adjustments evoked by load force perturbations of a grasped object. J Neurophysiol 60:1513-1522. Medline

Cothros N, Wong JD, Gribble PL (2006) Are there distinct neural representations of object and limb dynamics? Exp Brain Res 173:689-697. CrossRef Medline

Crevecoeur F, Thonnard JL, Lefèvre P (2010) Sensorimotor mapping for anticipatory grip force modulation. J Neurophysiol 104:1401-1408. CrossRef Medline

Criscimagna-Hemminger SE, Donchin O, Gazzaniga MS, Shadmehr R (2003) Learned dynamics of reaching movements generalize from dominant to nondominant arm. J Neurophysiol 89:168-176. Medline

Danion F (2007) The contribution of non-digital afferent signals to grip force adjustments evoked by brisk unloading of the arm or the held object. Clin Neurophysiol 118:146-154. CrossRef Medline

Danion F, Diamond JS, Flanagan JR (2012) The role of haptic feedback when manipulating nonrigid objects. J Neurophysiol 107:433-441. CrossRef Medline

Davidson PR, Wolpert DM, Scott SH, Flanagan JR (2005) Common encoding of novel dynamic loads applied to the hand and arm. J Neurosci 25:5425-5429. CrossRef Medline

Diedrichsen J, Hashambhoy Y, Rane T, Shadmehr R (2005) Neural correlates of reach errors. J Neurosci 25:9919-9931. CrossRef Medline

Flanagan JR, Wing AM (1997) The role of internal models in motion planning and control: evidence from grip force adjustments during movements of hand-held loads. J Neurosci 17:1519-1528. Medline

Flanagan JR, Vetter P, Johansson RS, Wolpert DM (2003) Prediction precedes control in motor learning. Curr Biol 13:146-150. CrossRef Medline

Flanagan JR, Bowman MC, Johansson RS (2006) Control strategies in object manipulation tasks. Curr Opin Neurobiol 16:650-659. CrossRef Medline

Goodbody SJ, Wolpert DM (1998) Temporal and amplitude generalization in motor learning. J Neurophysiol 79:1825-1838. Medline

Häger-Ross C, Johansson RS (1996) Nondigital afferent input in reactive control of fingertip forces during precision grip. Exp Brain Res 110: 131-141. Medline

Hogan N, Flash T (1987) Moving gracefully: quantitative theories of motor coordination. Trends Neurosci 10:170-173. CrossRef

Howard IS, Ingram JN, Wolpert DM (2009) A modular planar robotic manipulandum with end-point torque control. J Neurosci Methods 181:199-211. CrossRef Medline

Ingram JN, Howard IS, Flanagan JR, Wolpert DM (2010) Multiple graspspecific representations of tool dynamics mediate skillful manipulation. Curr Biol 20:618-623. CrossRef Medline

Ingram JN, Howard IS, Flanagan JR, Wolpert DM (2011) A single-rate context-dependent learning process underlies rapid adaptation to familiar object dynamics. PLoS Comput Biol 7:e1002196. CrossRef Medline

Izawa J, Rane T, Donchin O, Shadmehr R (2008) Motor adaptation as a process of reoptimization. J Neurosci 28:2883-2891. CrossRef Medline

Johansson RS, Flanagan JR (2009) Coding and use of tactile signals from the fingertips in object manipulation tasks. Nat Rev Neurosci 10:345-359. CrossRef Medline

Joiner WM, Ajayi O, Sing GC, Smith MA (2011) Linear hypergeneralization of learned dynamics across movement speeds reveals anisotropic, gainencoding primitives for motor adaptation. J Neurophysiol 105:45-59. CrossRef Medline

Kluzik J, Diedrichsen J, Shadmehr R, Bastian AJ (2008) Reach adaptation: what determines whether we learn an internal model of the tool or adapt the model of our arm? J Neurophysiol 100:1455-1464. CrossRef Medline

Lackner JR, DiZio P (2005) Motor control and learning in altered dynamic environments. Curr Opin Neurobiol 15:653-659. CrossRef Medline

Mah CD, Mussa-Ivaldi FA (2003) Generalization of object manipulation skills learned without limb motion. J Neurosci 23:4821-4825. Medline

Malfait N, Ostry DJ (2004) Is interlimb transfer of force-field adaptation a cognitive response to the sudden introduction of load? J Neurosci 24: 8084-8089. CrossRef Medline

Malfait N, Shiller DM, Ostry DJ (2002) Transfer of motor learning across arm configurations. J Neurosci 22:9656-9660. Medline

Mazzoni P, Krakauer JW (2006) An implicit plan overrides an explicit strategy during visuomotor adaptation. J Neurosci 26:3642-3645. CrossRef Medline

Melendez-Calderon A, Masia L, Gassert R, Sandini G, Burdet E (2011) Force field adaptation can be learned using vision in the absence of proprioceptive error. IEEE Trans Neural Syst Rehabil Eng 19:298-306. CrossRef Medline

Morasso P (1981) Spatial control of arm movements. Exp Brain Res 42:223227. Medline

Nowak DA, Hermsdörfer J, Schneider E, Glasauer S (2004) Moving objects in a rotating environment: rapid prediction of Coriolis and centrifugal force perturbations. Exp Brain Res 157:241-254. Medline

Ruitenbeek JC (1984) Invariants in loaded goal directed movements. Biol Cybern 51:11-20. CrossRef Medline

Scheidt RA, Reinkensmeyer DJ, Conditt MA, Rymer WZ, Mussa-Ivaldi FA (2000) Persistence of motor adaptation during constrained, multi-joint, arm movements. J Neurophysiol 84:853-862. Medline

Shadmehr R, Moussavi ZM (2000) Spatial generalization from learning dynamics of reaching movements. J Neurosci 20:7807-7815. Medline 
Shadmehr R, Mussa-Ivaldi FA (1994) Adaptive representation of dynamics during learning of a motor task. J Neurosci 14:3208-3224. Medline

Shadmehr R, Smith MA, Krakauer JW (2010) Error correction, sensory prediction, and adaptation in motor control. Annu Rev Neurosci 33:89-108. CrossRef Medline

Smith MA, Ghazizadeh A, Shadmehr R (2006) Interacting adaptive processes with different timescales underlie short-term motor learning. PLoS Biol 4:e179. CrossRef Medline
Thoroughman KA, Shadmehr R (2000) Learning of action through adaptive combination of motor primitives. Nature 407:742-747. CrossRef Medline

Wing AM, Flanagan JR, Richardson J (1997) Anticipatory postural adjustments in stance and grip. Exp Brain Res 116:122-130. CrossRef Medline

Wolpert DM, Diedrichsen J, Flanagan JR (2011) Principles of sensorimotor learning. Nat Rev Neurosci 12:739-751. Medline 